\title{
Desarrollo y estandarización de métodos de calibración para equipos utilizados en salud visual (Queratómetros, Lensómetros y Tonómetros), implementados en el Hospital Universitario de San Vicente Fundación
}

\author{
Olga Tobón, Victor Rodríguez \\ Instituto de Metrología Biomédica, Hospital Universitario de San Vicente Fundación, \\ Medellín, Colombia.
}

\begin{abstract}
Resumen -En el campo de la salud visual se utilizan una serie de equipos que ayudan al diagnóstico de la patología del paciente, pero actualmente no existe un método de calibración estandarizado para asegurar las mediciones que se realizan con estos dispositivos. El objetivo era estandarizar métodos de calibración para algunos de los equipos utilizados en el campo de la salud visual como son: queratómetros, lensómetros y tonómetros. Para la calibración de los tres tipos de equipos incluidos en este desarrollo, se utilizaron métodos de comparación directa de la indicación con la magnitud de los patrones utilizados que tenían trazabilidad con laboratorios nacionales e internacionales acreditados bajo la Norma NTC-ISO/IEC 17025:2005. Las mediciones realizadas por cada uno de los técnicos a los diferentes tipos de equipos en condiciones de repetibilidad y utilizando el método adoptado, se hicieron estadísticamente con la herramienta Anova Simple de Statgraphics, arrojando resultados satisfactorios con un valor-P por encima de 0,05 , igualmente se realizaron pruebas de exactitud, linealidad y robustez con resultados positivos. Los métodos adoptados fueron exitosamente validados y posteriormente estandarizados bajo la acreditación en la Norma NTC-ISO/ IEC 17025:2005.
\end{abstract}

Palabras clave - Calibración, validación, dioptrías, astigmatismo, presión intraocular, linealidad, exactitud, robustez.

\section{Development and standardization of Calibration Methods for Equipment Used in Visual Health (Keratometers, Lensometers and Tonometers), imPlemented in the Hospital Universitario de SAN Vicente Fundación}

\footnotetext{
Abstract - In the field of visual health, a series of equipment is used to aid in the diagnosis and treatment of patient pathology. However, no standardized calibration method to ensure the measurements performed with these devices exists today. The objective of the paper was to standardize calibration methods for some of the equipment used in the field of visual health such as keratometers,
}

\% Dirección para correspondencia: revistabme@eia.edu.co

DOI: https://doi.org/10.24050/19099762.n22.2017.1179 
lensometers and tonometers. For the calibration of the three types of equipment included in this development, methods of direct comparison were used of the indication with the magnitude of the standards that had traceability with national and international laboratories accredited under the Standard NTC-ISO / IEC 17025: 2005. The measurements made by each of the technicians to the different types of equipment under repeatable conditions and using the adopted method were analyzed statistically with the simple Anova tool of STATGRAPHICS, yielding satisfactory results with a P-value above 0.05 . Tests of accuracy, linearity and robustness were also performed with positive results. The adopted methods were successfully validated and later standardized under the accreditation in the NTC-ISO / IEC 17025: 2005 Standard.

Keywords - Calibration, validation, diopters, astigmatism, intraocular pressure, linearity, accuracy, robustness.

\title{
DESENVOLVIMENTO E ESTANDARDIZAÇÃO DE MÉTODOS DE CALIBRAÇÃO PARA EQUIPAMENTOS UTILIZADOS EM SAÚDE VISUAL IMPLEMENTADOS NO HOSPITAL UNIVERSITÁRIO DE SAN ViCENTE FUNDACIÓN
}

\begin{abstract}
Resumo-No campo da saúde visual utilizam-se uma série de equipas que ajudam ao diagnóstico da patologia do paciente, mas, atualmente não existe um método de calibração padronizado para assegurar as medidas que se realizam com estes dispositivos. O objetivo foi estandardizar métodos de calibração para alguns das equipas utilizadas no campo da saúde visual como são: ceratômetro, lenteômetros e tonometros. Para a calibração dos três tipos de equipamentos incluídos neste desenvolvimento, utilizaram-se métodos de comparação direta da indicação com a magnitude dos padrões utilizados que tinham rastreabilidade com laboratórios nacionais e internacionais acreditados baixo a Norma NTC-ISO/IEC 17025:2005. As medidas realizadas por cada um dos técnicos aos diferentes tipos de equipamentos em condições de repetir-se e utilizando o método adoptado, se analisaram estatisticamente com a ferramenta anova simples de statgraphics, produzindo resultados satisfatórios com um valor-P acima de 0,05, igualmente se realizaram provas de exatidão linearidade e robustez com resultados positivos. Os métodos adoptados foram validados de maneira exitosa e posteriormente padronizados baixo a acreditação na Norma NTC-ISO/ IEC 17025:2005.
\end{abstract}

Palavras-chave —Calibração, validação, dioptrias, astigmatismo, pressão intraocular, linearidade, exatidão, robustez.

\section{INTRODUCCIÓN}

$\mathrm{E}^{\mathrm{n}}$ n el campo de la salud visual, específicamente en el área de optometría y oftalmología se utilizan una serie de equipos como Queratómetros y Tonómetros para diagnóstico y Lensómetros para formulación de lentes, estos equipos se convierten en parte fundamental para el especialista que le permiten llegar a la toma de decisiones y son una herramienta necesaria para entregar al paciente un diagnostico asertivo y una formulación precisa y confiable.

De acuerdo a la aplicación clínica de estos equipos y la importancia que tienen en el momento de la emisión de un resultado, surge la necesidad de su calibración, que con el reporte de errores e incertidumbres en los puntos evaluados, ayuda al especialista a entregar diagnósticos más precisos y confiables; sin embargo actualmente no existe un método de calibración estandarizado para la calibración de estos equipos.

En virtud de lo anterior se desarrollaron tres métodos de calibración para cada uno de los equipos en evaluación (Queratómetro, tonómetro y lensometro), utilizando para los tres, técnicas de comparación directa con patrones trazables, en este contexto, el objetivo final aquí propuesto, fue la validación de estos métodos desarrollados y posteriormente la estandarización, mediante la acreditación bajo la norma NTC-ISO/IEC 17025:2005.

\section{MetodologíA}

Luego de un estudio de las especificaciones técnicas, modos de funcionamiento y uso, principios físicos ó fenómeno físico asociado al equipo ó magnitud a calibrar, se definió la metodología para desarrollar un procedimiento de calibración técnicamente apto que se pudiera modificar ó ajustar a las necesidades.

La estandarización de los métodos de calibración se desarrolló utilizando métodos de comparación directa de la indicación que reportaba cada equipo en evaluación con respecto a la magnitud de los patrones utilizados, que contaban con trazabilidad de laboratorios acreditados en la norma NTC-ISO/IEC 17025:2005.

Para los Queratómetros se utilizo un juego de esferas con unos diámetros correspondientes al intervalo de Dioptrías que se encuentran en el promedio de lecturas registradas normalmente por este equipo, equivalentes a la curvatura del ojo humano (40,50 D; 42,25 D; 45,00 D). En el caso de los tonómetros se utilizo un juego de cuatro 
pesas no normalizadas equivalente cada una a un valor de presión intraocular de $20 \mathrm{mmHg}$, que representa la fuerza que se utiliza para aplanar la cornea. Para los Lensómetros se utilizo un juego de lentes esféricos y uno cilíndrico que cubre el intervalo de trabajo normal de este equipo $(-20 \mathrm{D}$ a +20 D). La muestra para cada clase de dispositivo fue de tres diferentes tipos de equipo, que incluyeran indicación análoga o digital y que se encontraran normalmente dentro de las marcas comerciales.

Para la validación del método se contó con la participación de cuatro (4) metrólogos y cada uno de ellos realizo cinco (5) calibraciones para cada uno de los tres (3) diferentes tipos de equipos seleccionados, estas mediciones se realizaron en condiciones de repetibilidad, controlando las magnitudes de influencia que podrían afectar la prueba, como es el caso de la temperatura ambiente, que se estableció en $20^{\circ} \mathrm{C} \pm 2{ }^{\circ} \mathrm{C}$.

Para las pruebas de robustez del método se realizaron pruebas en sitios con diferentes alturas sobre el nivel del mar, donde además de la temperatura, cambia también la temperatura.

Las incertidumbres asociadas a cada método, fueron estudiadas y seleccionadas de acuerdo a normas de referencia que estaban relacionadas con las magnitudes de los diferentes equipos en el caso del Tonómetro, presión, fuerza y masa $(6,7,8)$ y en el caso de los Queratómetros y Lensómetros la magnitud dimensional (9).

En el análisis de los resultados se utilizó la prueba de Anova con el fin de buscar diferencia entre la media de cada punto evaluado entre un nivel de metrólogos y otro. Se consideraron diferencias estadísticas de $\mathrm{p}<0,05$ para la razón-F con un nivel de confianza del 95\%.

Para determinar la linealidad del método de calibración se utilizó la prueba de t-student. Se consideraron diferencias estadísticas para una $\mathrm{p}<0,05$.

\section{RESUltAdos}

Los resultados emitidos por cada metrólogo a través del método de comparación directa con los patrones de trabajo, para cada uno de los equipos evaluados, fueron analizados utilizando como método estadístico Anova simple, para determinar la repetibilidad y reproducibilidad, mediante la herramienta de STATGRAPHICS. La tabla ANOVA descompone la varianza de cada punto evaluado en dos componentes: un componente entre-grupos y un componente dentro-de-grupos (Intra grupos). La razón-F, es el cociente entre el estimado entre-grupos y el estimado dentro-de-grupos, puesto que el valor-P de la razón-F es mayor o igual que 0,05 , no existe una diferencia estadísticamente significativa entre la media de cada punto evaluado entre un nivel de metrólogos y otro, con un nivel de confianza del 95,0\%. Los resultados se encuentran en las Tablas 1,2 y 3 .

Tabla 1. Tabla Anova. Resultado de las mediciones para los tres Tonómetros

\begin{tabular}{cccc}
\hline Equipo & $\begin{array}{c}\text { Punto evaluado } \\
\text { (mmHg) }\end{array}$ & Razón-F & Valor-P \\
\hline Tonómetro & 20 & 1.18 & 0.3500 \\
Marca: Luxury & 40 & 0.02 & 0.9999 \\
Serie: 4254 & 60 & 0.02 & 0.9998 \\
& 80 & 0.04 & 0.9992 \\
\hline Tonómetro & 20 & 0.16 & 0.9246 \\
Marca: Zeiss & 40 & 1.52 & 0.2481 \\
Modelo: AT030 & 60 & 0.03 & 0.9923 \\
& 80 & 0.24 & 0.8655 \\
\hline Tonómetro & 20 & 0.55 & 0.6582 \\
Marca: Luxury & 40 & 0.04 & 0.9884 \\
Modelo: YX- & 60 & 0.56 & 0.6469 \\
30R & 80 & 0.47 & 0.7052 \\
\hline
\end{tabular}

Tabla 2. Tabla Anova. Resultado de las mediciones para los tres Queratómetros.

\begin{tabular}{cccc}
\hline Equipo & $\begin{array}{c}\text { Punto } \\
\text { evaluado } \\
\text { (D) }\end{array}$ & Razón-F & Valor-P \\
\hline Queratómetro Digital & 40,50 & 0.57 & 0.7209 \\
$\begin{array}{c}\text { Marca: Nidek } \\
\text { Modelo: ARK 500A } \\
\text { Serie: 530308 }\end{array}$ & 42,25 & 0.08 & 0.9947 \\
\hline Queratómetro Digital & 45,00 & 0.06 & 0.9968 \\
$\quad$ Marca: Nidek & 40,50 & 0.01 & 0.9993 \\
Modelo: ARK 500A & 42,25 & 0.17 & 0.9165 \\
$\quad$ Serie: 530265 & 45,00 & 0.01 & 0.9992 \\
\hline Queratómetro Análogo & 40,50 & 0.48 & 0.7000 \\
Marca: Topcon & 42,25 & 0.06 & 0.9784 \\
Modelo: OM-4 & 45,00 & 0.02 & 0.9952 \\
\hline Serie: 3026339 & & &
\end{tabular}

Tabla 3. Tabla Anova. Resultado de las mediciones para los tres Lensómetros

\begin{tabular}{cccc}
\hline Equipo & $\begin{array}{c}\text { Punto } \\
\text { evaluado (D) }\end{array}$ & Razón-F & Valor-P \\
\hline & +5 & 0.54 & 0.7425 \\
Lensómetro & -5 & 0.03 & 0.9996 \\
Digital & +10 & 0.11 & 0.9882 \\
Marca: Topcon & -10 & 0.04 & 0.9989 \\
Modelo: CL 200 & -13 & 1.09 & 0.3905 \\
Serie: 3903285 & +20 & 0.10 & 0.9912 \\
& -20 & 0.33 & 0.8909 \\
& 10 ( Cilindro ) & 0.09 & 0.9922 \\
\hline
\end{tabular}




\begin{tabular}{cccc}
\hline & +5 & 0.37 & 0.7770 \\
& -5 & 0.05 & 0.9837 \\
Lensómetro & +10 & 0.32 & 0.8113 \\
Digital & -10 & 0.51 & 0.6807 \\
Marca: Nidek & -13 & 0.04 & 0.9876 \\
Modelo: LM-500 & +20 & 0.10 & 0.9589 \\
Serie:404750 & -20 & 0.35 & 0.7923 \\
& $10($ Cilindro $)$ & 0.02 & 0.9970 \\
\hline Análogo & +5 & 0.24 & 0.8661 \\
Marca: Nidek & -5 & 0.03 & 0.9931 \\
Modelo: LM-770 & -10 & 0.05 & 0.9831 \\
Serie: 0503610 & +13 & 0.07 & 0.9739 \\
& -20 & 0.12 & 0.9483 \\
& $10($ Cilindro $)$ & 0.31 & 0.8211 \\
& & 0.14 & 0.9375 \\
\hline
\end{tabular}

$\mathrm{D}=$ Dioptrías

Por otro lado se evalúo la linealidad, exactitud y robustez del método, para determinar su validez. La linealidad permitió establecer la capacidad del método de calibración dentro de un intervalo determinado, de dar respuesta o resultados instrumentales que sean proporcionales al punto evaluado; cualitativamente el método es lineal evaluando el coeficiente de correlación $\mathrm{R}^{2}$ que es mayor a 0.99 , lo que significa que existe una correlación con una probabilidad elevada, sin embargo para obtener un mejor indicador lineal se utilizó una herramienta estadística de prueba t-student, obteniendo resultados de cumplimiento (10). Los resultados de estas pruebas se encuentran en las Tablas 4, 5 y 6 y las Figs. 1, 2 y 3.

Tabla 4. Resultados del promedio de los promedios de cada punto medido en los tres diferentes tonómetros, para determinar la linealidad del método de calibración mediante una prueba de t-student

\begin{tabular}{cccc}
\hline $\begin{array}{c}\text { PUNTOS } \\
\text { EVALUADOS } \\
\text { (mmHg) }\end{array}$ & $\begin{array}{c}\text { Tonómetro } \\
\text { Marca: } \\
\text { Luxury } \\
\text { Serie: } \mathbf{4 2 5 4}\end{array}$ & $\begin{array}{c}\text { Tonómetro } \\
\text { Marca: Zeiss } \\
\text { Modelo: AT030 }\end{array}$ & $\begin{array}{c}\text { Tonómetro } \\
\text { Marca: Luxury }\end{array}$ \\
\hline 20 & 20,03400 & 19,965 & 19,517 \\
\hline 40 & 40,08833 & 40,009 & 39,500 \\
\hline 60 & 60,53200 & 60,033 & 59,452 \\
\hline 80 & 81,11400 & 79,942 & 78,977 \\
\hline Pendiente & 1,01842 & 0,99978 & 0,99166 \\
\hline Intercepto & $-0,47884$ & $-0,00150$ & $-0,22150$ \\
\hline $\begin{array}{c}\text { Coeficiente de } \\
\text { Correlacion “ } \mathbf{r} \text { “ }\end{array}$ & 0,99998 & 1,00000 & 0,99998 \\
\hline $\begin{array}{c}\text { Coeficiente de } \\
\text { correlacion “ } \mathbf{R}^{2} \text { “ }\end{array}$ & 0,99996 & 1,00000 & 0,99997 \\
\hline t Cal & 238,788 & 893,556 & 255,446 \\
\hline t Critico & 6,205 & 6,205 & 6,205 \\
\hline $\begin{array}{c}\text { Criterio de } \\
\text { cumplimiento de } \\
\text { Linealidad }\end{array}$ & & $\mathrm{t}$ CAL $\geq \mathrm{t}$ CRITICO \\
\hline
\end{tabular}

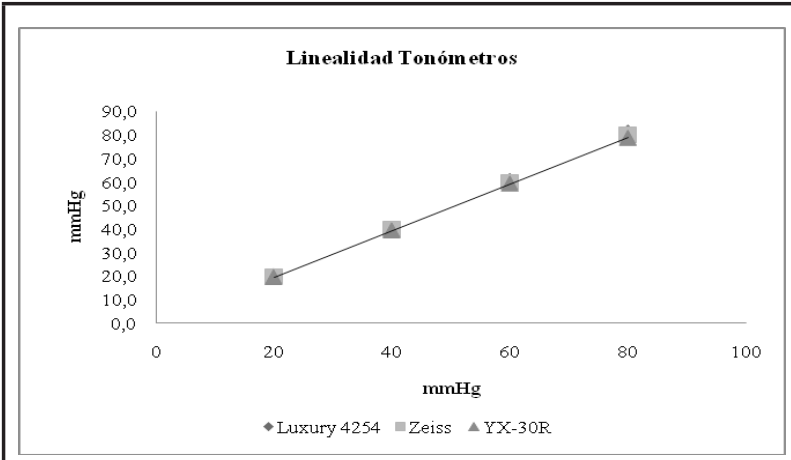

Fig. 1. Linealidad del método de calibración para tonómetros

Tabla 5. Resultados del promedio de los promedios de cada punto medido en los tres diferentes Queratómetros, para determinar la linealidad del método de calibración mediante una prueba de t-student

\begin{tabular}{|c|c|c|c|}
\hline $\begin{array}{c}\text { PUNTOS } \\
\text { EVALUADOS } \\
\text { (DIOPTRIAS) }\end{array}$ & $\begin{array}{c}\text { Queratómetro } \\
\text { Digital } \\
\text { Marca: Nidek } \\
\text { Modelo: ARK } \\
\text { 500A } \\
\text { Serie: } 530308\end{array}$ & $\begin{array}{c}\text { Queratómetro } \\
\text { Digital } \\
\text { Marca: Nidek } \\
\text { Modelo: ARK } \\
\text { 500A } \\
\text { Serie: 530265 }\end{array}$ & $\begin{array}{c}\text { Queratómetro } \\
\text { Análogo } \\
\text { Marca: Topcon } \\
\text { Modelo: OM-4 } \\
\text { Serie: } 3026339\end{array}$ \\
\hline 40,50 & 40,5026 & 40,63565 & 40,505 \\
\hline 42,25 & 42,15917 & 42,35625 & 42,2375 \\
\hline 45,00 & 45,00197 & 45,1491 & 45,01575 \\
\hline Pendiente & 1,00288 & 1,00411 & 1,00309 \\
\hline Intercepto & $-0,15129$ & $-0,04471$ & $-0,12888$ \\
\hline $\begin{array}{c}\text { Coeficiente de } \\
\text { Correlacion } \\
\text { " } r \text { " }\end{array}$ & 0,99972 & 0,99996 & 0,99999 \\
\hline $\begin{array}{c}\text { Coeficiente de } \\
\text { correlacion } \\
\text { “ } \mathbf{R}^{2} \text { “ } \\
\end{array}$ & 0,99945 & 0,99992 & 0,99997 \\
\hline t Cal & 42,63107 & 114,85658 & 183,27524 \\
\hline t Critico & 25,4517 & 25,4517 & 25,4517 \\
\hline
\end{tabular}

Criterio de
cumplimiento
de Linealidad $\quad \mathrm{t}$ cal $\leq \mathrm{t}$ critico

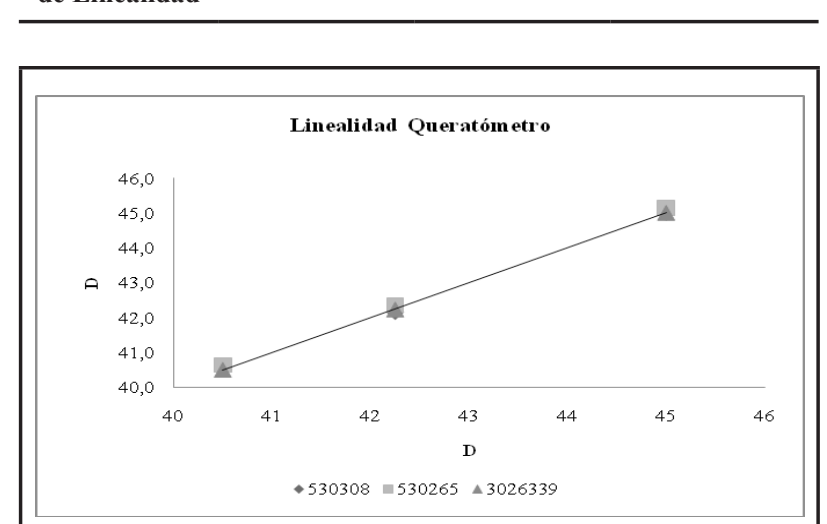

Fig. 2. Linealidad del método de calibración para Queratómetro 
Tabla 6. Resultados del promedio de los promedios de cada punto medido en los tres diferentes Lensómetros, para determinar la linealidad del método de calibración mediante una prueba de t-student

\begin{tabular}{|c|c|c|c|}
\hline $\begin{array}{c}\text { PUNTOS } \\
\text { EVALUADOS } \\
\text { (DIOPTRIAS) }\end{array}$ & $\begin{array}{c}\text { Lensómetro } \\
\text { Digital } \\
\text { Marca: Topcon } \\
\text { Modelo: CL } \\
\text { 200 } \\
\text { Serie: } \mathbf{3 9 0 3 2 8 5}\end{array}$ & $\begin{array}{c}\text { Lensómetro } \\
\text { Digital } \\
\text { Marca: } \\
\text { Nidek } \\
\text { Modelo: LM- } \\
\text { 500 } \\
\text { Serie:404750 }\end{array}$ & $\begin{array}{c}\text { Lensómetro } \\
\text { Análogo } \\
\text { Marca: Nidek } \\
\text { Modelo: LM- } \\
770 \\
\text { Serie: } \\
\text { 0503610 }\end{array}$ \\
\hline 5 & 5,0545 & 5,09351 & 5,02425 \\
\hline-5 & $-4,99653$ & $-5,0494$ & $-5,1115$ \\
\hline 10 & 10,38697 & 10,43582 & 10,40175 \\
\hline-10 & $-10,03333$ & $-10,14363$ & $-10,177$ \\
\hline$-13,3$ & $-13,28597$ & $-13,42956$ & $-13,42525$ \\
\hline 20 & 21,05327 & 21,0996 & 21,02975 \\
\hline-20 & $-19,88747$ & $-20,1256$ & $-20,1595$ \\
\hline 10 & 10,3226 & 10,40485 & 10,4125 \\
\hline Pendiente & 1,02045 & 1,02824 & 1,02759 \\
\hline Intercepto & 0,24769 & 0,20985 & 0,17326 \\
\hline $\begin{array}{c}\text { Coeficiente de } \\
\text { Correlacion } \\
\text { " } r \text { " }\end{array}$ & 0,99987 & 0,99991 & 0,99991 \\
\hline $\begin{array}{c}\text { Coeficiente de } \\
\text { correlacion } \\
\text { " } \mathbf{R}^{2} \text { “ }\end{array}$ & 0,99973 & 0,99982 & 0,99982 \\
\hline t Cal & 149,99988 & 181,36291 & 180,23781 \\
\hline t Critico & 2,96869 & 2,96869 & 2,96869 \\
\hline $\begin{array}{c}\text { Criterio de } \\
\text { cumplimiento } \\
\text { de Linealidad }\end{array}$ & \multicolumn{3}{|c|}{$\mathrm{t} \mathrm{CAL} \geq \mathrm{t}$ CRITICO } \\
\hline
\end{tabular}

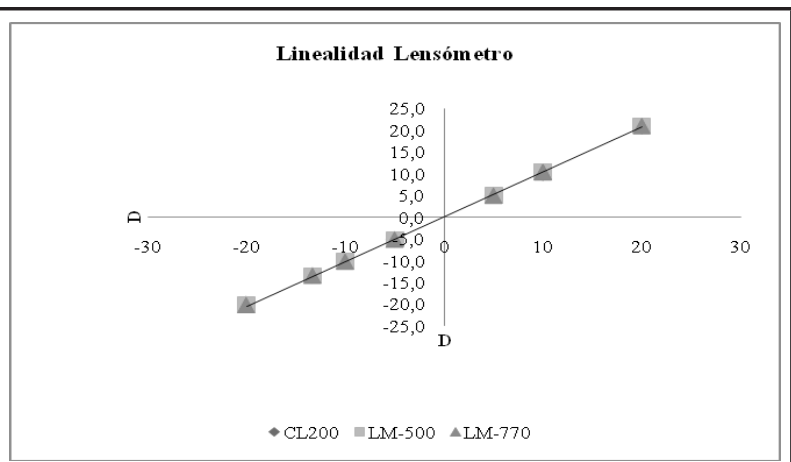

Fig. 3. Linealidad del método de calibración para Lensómetro

La exactitud esta aplicada a un conjunto de resultados de una calibración y supone una combinación de componentes aleatorios y un componente común de error sistemático o sesgo. Cuando se aplica a un método de calibración, el término "exactitud" se refiere a una combinación de veracidad y precisión. En esta prueba se determinó el grado de coincidencia existente entre el valor medio ob- tenido de una serie de resultados por cada metrólogo y un valor de referencia, se utilizó una herramienta estadística de prueba t- student (10). Los resultados se encuentran consignados en las Tablas 7, 8 y 9 .

Tabla 7. Resultados del promedio de los promedios de cada punto medido en los tres diferentes Tonómetros, para determinar la Exactitud del método de calibración mediante una prueba de t-student

\begin{tabular}{|c|c|c|c|c|c|}
\hline \multicolumn{2}{|c|}{ puntos evaluados (mmHg) } & \multirow{2}{*}{$\begin{array}{c}\mathbf{2 0} \\
20,03\end{array}$} & \multirow{2}{*}{$\begin{array}{c}\mathbf{4 0} \\
40,088\end{array}$} & \multirow{2}{*}{$\frac{\mathbf{6 0}}{60,532}$} & \multirow{2}{*}{$\begin{array}{c}\mathbf{8 0} \\
81,11\end{array}$} \\
\hline \multirow{7}{*}{$\begin{array}{l}\text { Tonómetro } \\
\text { Marca: } \\
\text { Luxury } \\
\text { Serie: } 4254\end{array}$} & $\begin{array}{l}\text { promedio de } \\
\text { los promedios } \\
\text { (mmHg) }\end{array}$ & & & & \\
\hline & $\begin{array}{l}\text { valor de } \\
\text { referencia } \\
(\mathrm{mmHg})\end{array}$ & 20 & 40 & 60 & 80 \\
\hline & sesgo $(\mathrm{mmHg})$ & 0,03 & 0,088 & 0,532 & 1,114 \\
\hline & $\begin{array}{c}\text { desviacion } \\
\text { estandar } \\
(\mathrm{mmHg})\end{array}$ & 0,119 & 0,08 & 0,21 & 0,323 \\
\hline & $\mathbf{n}$ & 20 & 20 & 20 & 20 \\
\hline & t cal & 0,05 & 0,2 & 0,46 & 0,63 \\
\hline & t critico & 2,36 & 2,36 & 2,36 & 2,36 \\
\hline \multirow{7}{*}{$\begin{array}{c}\text { Tonómetro } \\
\text { Marca: } \\
\text { Zeiss } \\
\text { Modelo: } \\
\text { AT030 }\end{array}$} & $\begin{array}{l}\text { promedio de } \\
\text { los promedios } \\
\text { (mmhg) }\end{array}$ & 19,965 & 40,018 & 60,033 & 79,94 \\
\hline & $\begin{array}{l}\text { valor de } \\
\text { referencia } \\
(\mathrm{mmHg})\end{array}$ & 20 & 40 & 60 & 80 \\
\hline & sesgo $(\mathrm{mmHg})$ & $-0,04$ & 0,018 & 0,033 & $-0,058$ \\
\hline & $\begin{array}{c}\text { desviacion } \\
\text { estandar } \\
(\mathrm{mmHg})\end{array}$ & 0,036 & 0,08 & 0,045 & 0,017 \\
\hline & $\mathbf{n}$ & 20 & 20 & 20 & 20 \\
\hline & t cal & $-0,22$ & 0,05 & 0,17 & $-0,76$ \\
\hline & t critico & 2,43 & 2,43 & 2,43 & 2,43 \\
\hline \multirow{7}{*}{$\begin{array}{c}\text { Tonómetro } \\
\text { Marca: } \\
\text { Luxury } \\
\text { Modelo: } \\
\text { YX-30R }\end{array}$} & $\begin{array}{l}\text { promedio de } \\
\text { los promedios } \\
(\mathrm{mmHg})\end{array}$ & 19,517 & 39,5 & 59,452 & 78,98 \\
\hline & $\begin{array}{l}\text { valor de } \\
\text { referencia } \\
(\mathrm{mmHg})\end{array}$ & 20 & 40 & 60 & 80 \\
\hline & sesgo $(\mathrm{mmHg})$ & $-0,48$ & $-0,5$ & $-0,548$ & $-1,023$ \\
\hline & $\begin{array}{c}\text { desviacion } \\
\text { estandar } \\
(\mathrm{mmHg})\end{array}$ & 0,018 & 0,033 & 0,036 & 0,039 \\
\hline & $\mathbf{n}$ & 20 & 20 & 20 & 20 \\
\hline & t cal & $-6,17$ & $-3,38$ & $-3,37$ & $-5,94$ \\
\hline & t critico & 2,43 & 2,43 & 2,43 & 2,43 \\
\hline \multicolumn{2}{|c|}{ Criterio de cumplimiento } & \multicolumn{4}{|c|}{$\mathrm{t}$ cal $\leq \mathrm{t}$ critico } \\
\hline
\end{tabular}


Tabla 8. Resultados del promedio de los promedios de cada punto medido en los tres diferentes Queratómetros, para determinar la Exactitud del método de calibración mediante una prueba de t-student

\begin{tabular}{|c|c|c|c|c|}
\hline \multicolumn{2}{|c|}{ Puntos evaluados (D) } & 40,50 & 42,25 & 45,00 \\
\hline \multirow{7}{*}{$\begin{array}{l}\text { Queratómetro } \\
\text { Digital } \\
\text { Marca: Nidek } \\
\text { Modelo: ARK } \\
\text { 500A } \\
\text { Serie: } 530308\end{array}$} & $\begin{array}{c}\text { promedio de los } \\
\text { promedios (D) }\end{array}$ & 40,5026 & 42,159 & 45,002 \\
\hline & $\begin{array}{c}\text { valor de } \\
\text { referencia (D) }\end{array}$ & 40,4936 & 42,186 & 45,02 \\
\hline & sesgo (D) & 0,009 & $-0,027$ & $-0,0193$ \\
\hline & $\begin{array}{c}\text { desviación } \\
\text { estándar (D) }\end{array}$ & 0,002 & 0,103 & 0,002 \\
\hline & $\mathbf{n}$ & 20 & 20 & 20 \\
\hline & t cal & 0,99 & 0,05 & 2,23 \\
\hline & t critico & 2,36 & 2,36 & 2,36 \\
\hline \multirow{7}{*}{$\begin{array}{l}\text { Queratómetro } \\
\text { Digital } \\
\text { Marca: Nidek } \\
\text { Modelo: ARK } \\
\text { 500A } \\
\text { Serie: } 530265\end{array}$} & $\begin{array}{c}\text { promedio de los } \\
\text { promedios (D) }\end{array}$ & 40,63565 & 42,356 & 45,1491 \\
\hline & $\begin{array}{c}\text { valor de } \\
\text { referencia (D) }\end{array}$ & 40,4936 & 42,186 & 45,02 \\
\hline & sesgo (D) & 0,142 & 0,1701 & 0,1278 \\
\hline & $\begin{array}{l}\text { desviacion } \\
\text { estandar (D) }\end{array}$ & 0,074 & 0,017 & 0,065 \\
\hline & $\mathbf{n}$ & 20 & 20 & 20 \\
\hline & $t$ cal & 0,43 & 2,2 & 0,44 \\
\hline & $t$ critico & 2,43 & 2,43 & 2,43 \\
\hline \multirow{7}{*}{$\begin{array}{c}\text { Queratómetro } \\
\text { Análogo } \\
\text { Marca: } \\
\text { Topcon } \\
\text { Modelo: } \\
\text { OM-4 } \\
\text { Serie: } \\
3026339\end{array}$} & $\begin{array}{c}\text { promedio de los } \\
\text { promedios (D) }\end{array}$ & 40,505 & 42,238 & 45,0158 \\
\hline & $\begin{array}{c}\text { valor de } \\
\text { referencia (D) }\end{array}$ & 40,4936 & 42,186 & 45,02 \\
\hline & sesgo (D) & 0,0114 & 0,0513 & $-0,0055$ \\
\hline & $\begin{array}{c}\text { desviación } \\
\text { estándar (D) }\end{array}$ & 0,01 & 0,013 & 0,008 \\
\hline & $\mathbf{n}$ & 20 & 20 & 20 \\
\hline & $t$ cal & 0,27 & 0,9 & 0,15 \\
\hline & $t$ critico & 2,43 & 2,43 & 2,43 \\
\hline \multicolumn{2}{|c|}{ criterio de cumplimiento } & \multicolumn{3}{|c|}{$\mathrm{t}$ cal $\leq \mathrm{t}$ critico } \\
\hline
\end{tabular}

Tabla 9. Resultados del promedio de los promedios de cada punto medido en los tres diferentes Lensómetros, para determinar la Exactitud del método de calibración mediante una prueba de t-student

\begin{tabular}{|c|c|c|c|c|c|c|}
\hline \multicolumn{2}{|c|}{ Puntos evaluados (D) } & 5,00 & -5 & -10 & 20 & 10 \\
\hline \multirow{7}{*}{$\begin{array}{c}\text { Lensómetro } \\
\text { Digital } \\
\text { Marca: } \\
\text { Topcon } \\
\text { Modelo: } \\
\text { CL 200 } \\
\text { Serie: } \\
3903285\end{array}$} & $\begin{array}{l}\text { promedio de } \\
\text { los promedios } \\
\text { (D) }\end{array}$ & 5,05 & $-5,00$ & $-10,03$ & 21,05 & 10,32 \\
\hline & $\begin{array}{c}\text { valor de } \\
\text { referencia (D) }\end{array}$ & 5,07 & $-5,05$ & $-10,16$ & 21,07 & 10,41 \\
\hline & sesgo (D) & $-0,02$ & 0,00 & 0,07 & $-0,02$ & $-0,08$ \\
\hline & $\begin{array}{c}\text { desviacion } \\
\text { estandar (D) }\end{array}$ & 0,00 & 0,00 & 0,01 & 0,01 & 0,01 \\
\hline & $\mathbf{n}$ & 20,00 & 20,00 & 20,00 & 20,00 & 20,00 \\
\hline & t cal & 2,10 & 0,32 & 2,14 & 0,42 & 2,35 \\
\hline & t critico & 2,36 & 2,36 & 2,36 & 2,36 & 2,36 \\
\hline
\end{tabular}

\begin{tabular}{|c|c|c|c|c|c|c|}
\hline \multirow{7}{*}{$\begin{array}{c}\text { Lensómetro } \\
\text { Digital } \\
\text { Marca: } \\
\text { Nidek } \\
\text { Modelo: } \\
\text { LM-500 } \\
\text { Serie: } \\
\text { 404750 }\end{array}$} & $\begin{array}{l}\text { promedio de } \\
\text { los promedios } \\
\text { (D) }\end{array}$ & 5,09 & $-5,05$ & $-10,14$ & 21,10 & 10,40 \\
\hline & $\begin{array}{c}\text { valor de } \\
\text { referencia (D) }\end{array}$ & 5,07 & $-5,05$ & $-10,16$ & 21,07 & 10,41 \\
\hline & sesgo (D) & 0,02 & 0,00 & 0,02 & 0,03 & 0,00 \\
\hline & $\begin{array}{c}\text { desviación } \\
\text { estándar (D) }\end{array}$ & 0,01 & 0,00 & 0,01 & 0,00 & 0,01 \\
\hline & $\mathbf{n}$ & 20,00 & 20,00 & 20,00 & 20,00 & 20,00 \\
\hline & t cal & 0,84 & 0,30 & 0,66 & 1,72 & 0,09 \\
\hline & t critico & 2,43 & 2,43 & 2,43 & 2,43 & 2,43 \\
\hline \multirow{7}{*}{$\begin{array}{c}\text { Lensómetro } \\
\text { Análogo } \\
\text { Marca: } \\
\text { Nidek } \\
\text { Modelo: } \\
\text { LM-770 } \\
\text { Serie: } \\
\text { 0503610 }\end{array}$} & $\begin{array}{l}\text { promedio de } \\
\text { los promedios } \\
\text { (D) }\end{array}$ & 5,02 & $-5,11$ & $-10,18$ & 21,03 & 10,41 \\
\hline & $\begin{array}{c}\text { valor de } \\
\text { referencia (D) }\end{array}$ & 5,07 & $-5,05$ & $-10,16$ & 21,07 & 10,41 \\
\hline & sesgo (D) & $-0,05$ & $-0,07$ & $-0,02$ & $-0,04$ & 0,01 \\
\hline & $\begin{array}{c}\text { desviación } \\
\text { estándar (D) }\end{array}$ & 0,01 & 0,02 & 0,02 & 0,02 & 0,02 \\
\hline & n & 20,00 & 20,00 & 20,00 & 20,00 & 20,00 \\
\hline & t cal & 1,04 & 0,95 & 0,24 & 0,44 & 0,08 \\
\hline & $t$ critico & 2,43 & 2,43 & 2,43 & 2,43 & 2,43 \\
\hline
\end{tabular}

Criterio de cumplimiento

$\mathrm{t}$ cal $\leq \mathrm{t}$ critico

$\mathrm{D}=$ Dioptrias

La robustez es una medida de la capacidad de un procedimiento de calibración de no ser afectado por variaciones pequeñas pero deliberadas de los parámetros del método; proporciona una indicación de la fiabilidad del procedimiento en un uso normal. En este sentido el objetivo de la prueba de robustez es optimizar el método de calibración desarrollado por el laboratorio y describir bajo qué condiciones analíticas (incluidas sus tolerancias), se pueden obtener a través de estos, resultados confiables. Las pruebas fueron realizadas en dos diferentes ambientes donde las condiciones ambientales de temperatura y presión barométrica son extremas, determinando para cada variación si es sensible para el método desarrollado (10).

Los métodos desarrollados fueron acreditados bajo la norma NTC-ISO/IEC 17025:2005 "Requisitos generales para la Competencia de los Laboratorios de Ensayo y Calibración" por el Organismo Nacional de Acreditación en Colombia ONAC. El alcance de la acreditación se encuentra resumido en la Tabla 10. 
Tabla 10. Alcance de la acreditación bajo la Norma NTC-ISO/IEC 17025:2005

\begin{tabular}{|c|c|c|c|}
\hline Magnitud & $\begin{array}{l}\text { Intervalo de } \\
\text { medición }\end{array}$ & CMC & $\begin{array}{c}\text { Instrumentos a } \\
\text { calibrar }\end{array}$ \\
\hline \multirow{2}{*}{$\begin{array}{l}\text { Radio de } \\
\text { curvatura- } \\
\text { poder } \\
\text { dióptrico }\end{array}$} & $\begin{array}{l}(40,50 \text { A } 45,00) \mathrm{D} \\
(7,54 \text { A } 8,33) \mathrm{mm}\end{array}$ & $\begin{array}{l} \pm 0,0069 \mathrm{D} \\
\pm 1,1 \mu \mathrm{m}\end{array}$ & $\begin{array}{c}\text { Queratometro } \\
\text { digital, } \\
\text { Autorefractoquer } \\
\text {-atometro. } \\
\text { En sitio y en las } \\
\text { instalaciones del } \\
\text { laboratorio }\end{array}$ \\
\hline & $\begin{array}{l}(40,50 \text { A } 45,00) \mathrm{D} \\
(7,54 \text { A } 8,33) \mathrm{mm}\end{array}$ & $\begin{array}{l} \pm 0,018 \mathrm{D} \\
\pm 3,0 \mu \mathrm{m}\end{array}$ & $\begin{array}{l}\text { Queratometro } \\
\quad \text { análogo } \\
\text { En sitio y en las } \\
\text { instalaciones del } \\
\quad \text { laboratorio }\end{array}$ \\
\hline \multirow{2}{*}{$\begin{array}{l}\text { Poder } \\
\text { dióptrico }\end{array}$} & $\begin{array}{l}\text { ESFERAS }( \pm 5 \mathrm{~A} \pm \\
\text { 20) D CILINDROS } \\
10 \mathrm{D}\end{array}$ & $\pm 0,0086 \mathrm{D}$ & $\begin{array}{l}\text { lensómetro digital } \\
\text { En sitio y en las } \\
\text { instalaciones del } \\
\text { laboratorio }\end{array}$ \\
\hline & $\begin{array}{l}\text { ESFERAS }( \pm 5 \mathrm{~A} \pm \\
\text { 20) D CILINDROS } \\
10 \mathrm{D}\end{array}$ & $\pm 0,022 \mathrm{D}$ & $\begin{array}{c}\text { lensómetro análogo } \\
\text { En sitio y en las } \\
\text { instalaciones del } \\
\text { laboratorio }\end{array}$ \\
\hline $\begin{array}{l}\text { Presión } \\
\text { ocular }\end{array}$ & $\begin{array}{c}(20-80) \mathrm{mmHg} \\
(2,66-10,66) \mathrm{kPa}\end{array}$ & $\begin{array}{c} \pm 0,095 \\
\mathrm{mmHg} \\
\pm 0,013 \mathrm{kPa}\end{array}$ & $\begin{array}{l}\text { TONOMETRO DE } \\
\text { GOLDMAN (con } \\
\text { sistema de peaje) } \\
\text { En sitio y en las } \\
\text { instalaciones del } \\
\text { laboratorio }\end{array}$ \\
\hline
\end{tabular}

\section{Discusión}

Se desarrollaron unos métodos para la calibración de tonómetros, lensómetros y Queratómetros, basados en la comparación directa de las lecturas con patrones trazables, logro de suma importancia para el crecimiento de Colombia en el contexto de la metrología, que además genera reconocimiento en más de 85 países firmantes de los acuerdos multilaterales gracias al otorgamiento de la acreditación bajo la Norma NTC- ISO/IEC 17025:2005 recibido por parte de el Organismo Nacional de Acreditación (ONAC).

Las incertidumbres estimadas para cada proceso de medición que están reflejadas en el alcance de la acreditación en la Tabla 10, fueron debidamente presupuestadas de acuerdo a un análisis previo y con el apoyo de varias referencias normativas, considerando algunas componentes que aportaban los equipos bajo prueba, otras aportadas por los equipos patrón utilizados para cada medición, y además la influencia del medio en el cual se estaban llevando a cabo las mediciones como es el caso de la temperatura, finalmente se reporta una incertidumbre expandida con un factor de cobertura $\mathrm{k}=2$, con un nivel de confianza del $95 \%$.

Al aplicar las pruebas de robustez, teniendo en consideración la exhaustiva revisión e inclusión de las compo- nentes de incertidumbre que podían presentar influencia en los resultados de las calibraciones, se obtuvo un método confiable ante variaciones de los parámetros que puedan afectar las mediciones.

Aunque la participación en pruebas interlaboratorios es uno de los elementos que se debe considerar para demostrar la validación de los métodos de calibración, en este caso no fue posible aplicarlo debido a que no se encontró ninguna oferta de los entes oficiales nacionales o internacionales para los ensayos de aptitud. Esto implicó que el ONAC, emitiera una carta de aceptación en la cual se especifica que esta prueba no es aplicable.

\section{Conclusión}

Después del tratamiento y análisis de los resultados de las mediciones realizadas en condiciones de repetibilidad a tres diferentes tipos de equipos por un equipo de metrólogos, se concluye que los métodos de calibración desarrollados son validos, debido a que la repetibilidad y reproducibilidad comprobada con el método Anova están dentro de los parámetros establecidos por la herramienta, como también se encuentra la linealidad la exactitud y la robustez del método.

El resultado de las pruebas de repetibilidad y reproducibilidad permitieron concluir que el método aplicado, los equipos patrón utilizados y el personal técnico involucrado cumplen con los parámetros necesarios para ser considerado un método estándar y garantizan el aseguramiento de la calidad de las mediciones.

El análisis estadístico de los resultados obtenidos en las calibraciones indica que el método es repetible y reproducible, que el personal es idóneo y que los equipos patrón son confiables.

\section{Agradecimientos}

A Colciencias por el patrocinio con este proyecto y por creer en nuestro trabajo y darnos esta oportunidad.

Al Hospital Universitario de San Vicente Fundación por el apoyo en todo el desarrollo del proyecto de investigación y en particular a la unidad de investigaciones, la unidad de oftalmología de pensionados, medicina física y rehabilitación.

A los colaboradores del instituto de metrología Biomédica por su trabajo comprometido y entusiasmo con este proyecto.

Al Dr. Amilcar Estrada por sus importantes aportes.

Al Dr. Esaú Astudillo por su apoyo y disposición en el desarrollo de esta investigación. 


\section{REFERENCIAS}

[1]. Romero Caballero MD. Descriptores cuantitativos de la topografía corneal. Intreduccion. Tesis Doctoral. Universidad de Murcia. Departamento de Oftalmologia. Febrero. 1998.

[2]. Eliot M. Kirstein, Ahmed Elsheik, Pinakin Gunvant. Capitulo 6 Tonometry Past, Present and Future. Glaucoma- Current Clinical and Research Aspects.

[3]. Luna Martínez I, Brechtel Bindel M, Fuente Torres MA. Relación del espesor corneal central y la variación en la presión intraocular con daño al nervio óptico en pacientes mexicanos con glaucoma. Rev Mex Oftalmol. 2009; 83(4):193-6.

[4]. Jiménez Rodríguez E, López de Cobos M, Luque Aranda R, López-Egea Bueno MA, Vázquez Salvi AI, García Campos JM. Correlación entre el grosor corneal central, presión intraocular y afectación glaucomatosa del campo visual. Arch Soc Esp Oftalmol. 2009;84(3):139-43

[5]. M. Martínez Corral, W. D. Furlan, A. Pons Marti y G. Saavedra Tortosa, Instrumentos ópticos y optométricos: Teoría y prácticas., Universitat de Valencia, España (1998).

[6]. DKD-R-6-1 Calibration of Pressure Gauges, Edition 01, 2003

[7]. Euramet/cg-04/v.01, Incertidumbre en Mediciones de Fuerza, $1^{\text {a }}$ Edicion digital de la traducción al español, Marzo 2010

[8]. Guia para la Calibracion de los Instrumentos para pesar de funcionamiento no automatico, SIM MWG7/cg-01/v.00, 2009

[9]. Guia Tecnica sobre Trazabilidad e incertidumbre en Metrologia Dimensional, EMA, Revision 1, Abril de 2008.

[10]. Guía Técnica No 1, “Aspectos Generales Sobre la Validación de Métodos", Instituto de Salud Publica, Santiago -Chile, 2010. 